\title{
Central venous catheterization in cancer patients with severe thrombocytopenia: Ultrasound-guide improves safety avoiding prophylactic platelet transfusion
}

\author{
LUIGI CAVANNA, CHIARA CITTERIO, CAMILLA DI NUNZIO, \\ ELENA ORLANDI, ILARIA TOSCANI and MASSIMO AMBROGGI \\ Department of Onco-Haematology, Hospital Guglielmo da Saliceto, I-29121 Piacenza, Italy \\ Received January 7, 2019; Accepted June 18, 2019
}

DOI: $10.3892 / \mathrm{mco} .2020 .2010$

\begin{abstract}
Prior research has revealed that ultrasound (US) guided central venous catheterization (CVC) is associated with a reduction in the complication rate such as pneumothorax and an improved first-pass success placing CVC in the internal jugular vein. The present study investigated if US-guided CVC, in a subset of cancer patients with severe thrombocytopenia, reduced bleeding risk and avoided prophylactic platelet transfusion. The efficacy and safety of US-guided CVC placement in cancer patients with severe thrombocytopenia was retrospectively analyzed over a period of 9 years (Dec 2000-Jan 2009), 1,660 and 207 patients with cancer underwent US-guided CVC placement into internal jugular vein respectively at the Department of Onco-Haematology, Hospital of Piacenza. The first group of patients included patients in active antitumor treatment, while the second group included patients in the palliative phase. A total of 110 (5.89\%) of these 1,867 patients exhibited severe thrombocytopenia defined as platelet count $\leq 20 \times 10^{9} / 1$, and formed the basis of this study. All procedures were evaluated for bleeding complications as defined by the National Institute of Health Common Terminology Criteria for Adverse Events (CTCAE 3.0). In the subgroup of the 110 patients with severe thrombocytopenia a single needle puncture of the vein was employed in 121 of the 122 procedures $(99.18 \%)$ and no attempt failures were
\end{abstract}

Correspondence to: Dr Luigi Cavanna, Department of Onco-Haematology, Hospital Guglielmo da Saliceto, Via Taverna 49, I-29121 Piacenza, Italy

E-mail: 1.cavanna@ausl.pc.it; luigicavanna53@gmail.com

Abbreviations: US, ultrasound; CVC, central venous catheterization; CTCAE, Common Terminology Criteria for Adverse Events; PICC, peripherally inserted central catheter; RBC, red blood cells; UK, United Kingdom

Key words: cancer, central venous catheter, thrombocytopenia, central venous catheterization registered. No pneumothorax, no major bleeding and no nerve and arterial puncture were reported, only one self-limiting hematoma $(0.90 \%)$ at the site of $\mathrm{CVC}$ insertion was reported (CTCAE 3.0 grade 1). No platelet transfusions were performed in the 110 patients, pre and post CVC placement. We believe that US-guided CVC insertion procedures into the internal jugular vein makes the difference in safety, also in thrombocytopenic patients avoiding prophylactic or post procedure platelet transfusion.

\section{Introduction}

Central venous catheters (CVC) is essential in patients with cancer, and the need for intravenous access devices for the administration of cancer therapy has increased proportionally with the increasing number of patients diagnosed with cancer. CVCs include implantable central venous ports (PORTS), peripherally inserted central catheters (PICCs) and external CVCs. The percutaneous approach to the subclavian or internal jugular vein is a popular procedure for placing catheters in the superior vena cava both for short-term and long-term use. Unfortunately, central venous catheter insertion into the jugular or subclavian vein represents a risk of pneumothorax, nerve puncture and major bleeding (mechanical complications), infection and CVC-related vein thrombosis $(1,2)$. Mechanical complications of CVC insertion without ultrasound (US) guidance, such as arterial puncture and pneumothorax, are seen in up to $21 \%$ of attempts, and up to $35 \%$ of insertion attempts are not successful (3-5). Prior researches show that the use of US-guide CVC has been associated with a reduction in complication rate and an improved first-pass success when placing CVC in the internal jugular vein (6-18). We sought to investigate within US-guidance CVC in a subset of cancer patients with severe thrombocytopenia also effects the safety, reducing bleeding complications and avoid prophylactic platelet transfusion. In this paper, we report clinical outcome on US-guided CVC insertional procedures performed in 1,867 cancer patients over a period of 9 years and we aim to evaluate the safety of US-guided CVC insertion in the internal jugular vein in a subgroup of cancer patients with severe thrombocytopenia defined as platelet count below $20 \times 10^{9} / 1(19)$. 


\section{Patients and methods}

Patients. This research was conducted based on the medical records of cancer patients to identify those who underwent US-guidance CVC placement in the internal jugular vein, and 1,867 consecutive patients were identified at the Department of Oncology-Haematology (Piacenza, Italy). All patients included in the study signed informed consent before undergoing the procedure; they signed also informed consent to utilize their clinical data for clinical research. These clinical data were abstracted from the medical records: Clinical setting: Age, diagnosis and sex. Indications for CVC insertion: Hemoglobin levels, white blood cell and platelet count in a sample obtained within 1 day prior to performing the procedure, chest radiographic findings pre- and post-CVC, pneumothorax, tube thoracotomy, hematoma, nerve puncture, hemorrhagic complication rate. Post-procedural hemoglobin values within $24 \mathrm{~h}$ and 3 days, packed RBC transfusion within 6 days after the procedure and platelet transfusion pre- and post-procedure were also recorded. Hemorrhagic complications were defined using the National Institutes of Health Common Terminology Criteria for Adverse Events (CTCAE 3.0) (20). Grade 1 bleeding is characterized by mild symptoms not requiring any intervention, for example, local hematoma. Grade 2 bleeding has mild symptoms requiring invasive interventions such as evacuation or aspiration. Grade 3 bleeding requires transfusion, radiologic, endoscopic or elective operative interventions. In grade 4 bleeding, life-threatening consequences make urgent intervention necessary. A total of 110 patients had severe thrombocytopenia defined as platelet count $\leq 20 \times 10^{9} / 1$ and they form the basis of this report.

$U S$-guided CVC. The procedure was performed using Esaote SpA equipped with two transducers between 3.5 to $7.5 \mathrm{MHZ}$, with a needle guide. The method that we commonly use is 'the three-handed method', as previously reported $(17,18)$; this method requires an assistant to hold the probe, while the operator controls the needle and performs the procedure under real-time guidance, and the nurse helps the two physicians during the maneuver. The central vein was identified along its greater longitudinal axis and its relationship with other anatomical structures using Valsalva's maneuver which determines an increase of the diameter of the veins. Under US-guide in real time, a 16-gauge needle is introduced into the last portion of internal jugular vein. This vein was reached through the transducer placed at the point of insertion of the sternocleidomastoid muscle into the clavicular; the correct introduction of the needle was always confirmed by US guidance and by the easy aspiration of venous blood.

The Seldinger technique was used to place the catheter, which was advanced into the superior vena cava until insertion into right atrium.

Every procedure was scheduled in order to register patient's data, pathological diagnosis, indications for CVC insertion, type of CVC, number of attempts and early complications if any failure. Medications, CVC-related blood stream infection, symptomatic deep-vein thrombosis and CVC removal or substitution were also recorded. Within $2 \mathrm{~h}$ after each procedure, chest radiography and US scanning were carried out to exclude pneumothorax and to evaluate correct catheter position.
Statistical analysis. Demographic data and clinical features were analyzed using descriptive methods. Quantitative variables were summarized using mean and standard deviation. Categorical variables were summarized as counts and percentages. Baseline analysis included all enrolled patients. Statistical tests were performed with Microsoft Excel 2010 software (Microsoft Corporation).

\section{Results}

Over a period of 9 years a total of 2,187 CVC insertional procedures were applied to 1,867 cancer patients that underwent US-guided CVC catheterization in internal jugular vein at the Department of Oncology-Haematology, Hospital Guglielmo da Saliceto (Table I).

Of these 1,867 patients, 1,660 were treated with anticancer therapy and underwent 1,978 CVC insertional US-guided procedures with these indications: Chemotherapy delivery, transfusion, parenteral nutrition, leukapheresis, autologous and allogenic stem cell transplantation, invasive hemodynamic variables assessment and blood sampling. In this group the procedure was performed in 380 patients with hematologic malignancies and in 1,280 patients with solid tumors; the majority of patients with solid tumors had gastrointestinal cancer and the majority of patients with hematologic malignancies had lymphomas. The median platelet count at the time of CVC insertion was $236 \times 10^{9} / 1$ (range 7-510x10 $/ 1$ ). The remaining 207 cancer patients were in advanced phase of their disease and underwent 209 US-guide CVC catheterization for parenteral nutrition and for hydration since they were in palliative phase of their trajectory of cancer history. The median platelet count at the time of CVC was $194 \times 10^{9} / 1$ (range $7-254 \times 10^{9} / 1$ ). The procedure was performed in 7 patients with hematologic malignancies and in 200 patients with solid tumors (Table I); the majority of patients with solid tumors had gastrointestinal cancer and the majority of patients with hematologic malignancies had lymphomas (Table I). In these two series including 1,867 patients with cancer that underwent 2,187 insertional procedure, 110 patients (5.89\%) had platelet count $\leq 20 \times 10^{9} / 1$ (Table II).

The median platelet count in this group was $12 \times 10^{9} / 1$ (range $\left.7-20 \times 10^{9} / 1\right)$, these 110 patients underwent 122 insertional procedures (Table II). In the entire group of 1,867 cancer patients that underwent 2,187 insertional CVC procedure, no pneumothorax, no major bleeding, no nerve puncture were reported, only 6 arterial puncture of 2,187 procedures $(0.27 \%)$ and $4(0.18 \%)$ of self-limiting hematomas were registered. In the subgroup of 110 patients with severe thrombocytopenia a single needle puncture of the vein was done on 121 of the 122 procedures $(99.18 \%)$ and not attempts failure were registered, no pneumothorax, no major bleeding and no nerve and arterial puncture were reported, only one self-limiting hematoma $(0.90 \%)$ at the site of CVC insertion was reported (CTCAE 3.0 grade 1). No platelet transfusion were done in the 110 patients, pre and post CVC placement.

\section{Discussion}

The use of central venous access devices has become an essential component of the treatment of many medical disorders. Central venous access is commonly attempted in 
Table I. Characteristics and results of ultrasound guided central catheter insertion in the internal jugular vein over a period of 9 years in 1,867 cancer patients.

\begin{tabular}{|c|c|c|}
\hline Characteristics & $\begin{array}{l}\text { Cancer patients in } \\
\text { antitumoral treatment, } \mathrm{n}(\%)\end{array}$ & $\begin{array}{c}\text { Cancer patients in } \\
\text { palliative phase, } \mathrm{n}(\%)\end{array}$ \\
\hline No. of patients total $(\%)$ & $1,660(100)$ & $207(100)$ \\
\hline Male & $858(52)$ & $106(51.2)$ \\
\hline Female & $802(48)$ & $101(48.8)$ \\
\hline Median age years (range) & $61.71(18-85)$ & $68(22-86)$ \\
\hline Type of cancer total $(\%)$ & $1,660(100)$ & $207(100)$ \\
\hline Solid tumor total $(\%)$ & $1,280(77.1)$ & $200(96.6)$ \\
\hline Hematological cancer total (\%) & $380(22.9)$ & $7(2.4)$ \\
\hline Platelet count $\times 10^{9} / 1$ median (range) & $236(7-510)$ & $194(7-254)$ \\
\hline Total procedures of catheter insertion (total \%) & $1,978(100)$ & $209(100)$ \\
\hline Access with one attempt & $1,948(98.5)$ & $206(98.6)$ \\
\hline Access with two attempt & $30(1.5)$ & $3(1.4)$ \\
\hline Failure total $(\%)$ & $18(0.9)$ & $0(0)$ \\
\hline Pneumothorax & $0(0)$ & $0(0)$ \\
\hline Major bleeding & $0(0)$ & $0(0)$ \\
\hline Mediastinal CVC dislocation & $2(11.1)$ & $0(0)$ \\
\hline Arterial puncture & $6(33.35)$ & $0(0)$ \\
\hline Vein collapse & $8(44.45)$ & $0(0)$ \\
\hline Nerve puncture & $0(0)$ & $0(0)$ \\
\hline No efficacious 'eco window' & $2(11.1)$ & $0(0)$ \\
\hline
\end{tabular}

Table II. Results of the ultrasound-guided central vein catheterization insertional procedure in the 110 thrombocytopenic cancer patients (PLTs count $\leq 20 \times 10^{9} / 1$; range $7-20 \times 10^{9} / 1$ ).

\begin{tabular}{|c|c|c|c|}
\hline Results & Total n (\%) & $\begin{array}{l}\text { Cancer patients in } \\
\text { antitumoral treatment, } \mathrm{n}(\%)\end{array}$ & $\begin{array}{c}\text { Cancer patients in } \\
\text { palliative phase, } \mathrm{n}(\%)\end{array}$ \\
\hline Total patients & $110(100)$ & $70(100)$ & $40(100)$ \\
\hline Total CVC insertional procedures & $122(100)$ & $80(100)$ & $42(100)$ \\
\hline Access with one attempt & $121(99.18)$ & $79(98.75)$ & $42(100)$ \\
\hline Access with two attempts & $1(0.82)$ & $1(1.25)$ & $0(0)$ \\
\hline Pneumothorax & $0(0)$ & $0(0)$ & $0(0)$ \\
\hline Major bleeding & $0(0)$ & $0(0)$ & $0(0)$ \\
\hline Arterial puncture & $0(0)$ & $0(0)$ & $0(0)$ \\
\hline Failure & $0(0)$ & $0(0)$ & $0(0)$ \\
\hline Nerve puncture & $0(0)$ & $0(0)$ & $0(0)$ \\
\hline Local hematoma & $1(0.82)$ & $1(1.25)$ & $0(0)$ \\
\hline
\end{tabular}

the internal jugular vein, subclavian vein, femoral vein, or arm veins using peripherally central catheters. It is estimated that several million devices are inserted each year, facilitating many emerging therapies, including long-term chemotherapy (21). Central venous cannulation can be unsafe: The National Confidential Enquiry into perioperative deaths has reported one death resulting from a procedure-induced pneumothorax (22).

In clinical practice a challenging clinical scenario can occur when urgent treatment is necessary in patients at high risk of bleeding because of an underlying onco-hematological disorder with a very low platelet count, furthermore, such as patients with acute leukemia, in these patients US-guidance CVC can allow and immediately adequate treatment (17). Traditionally, the site of central venous access is guided by anatomical landmarks such as bony prominences, muscle surfaces, and arterial pulsations. This 'blind' approach to the central veins assumes anatomical uniformity, does not account for the possibility of occlusions, and depends on correct discernment of the relationship among multiple anatomical landmarks. For these reasons, the procedure is associated with a relatively high incidence of complications, related to first pass failure, arterial punctures or pneumothorax (6-18). 
In comparison to standard CVC, US-guided CVC is more feasible and offers various advantages (ease of vein identification, a shorter duration of the procedure) which, together with a higher rate of success and decreased incidence of complications, make the latter preferable to the former, especially in high-risk patients.

Patients with a low platelet count, above all patients with cancer, often require the insertion of $\mathrm{CVC}$, and low platelet count may be considered a relative contraindication to the insertion of a CVC due to the risk of bleeding (23-28). Current practice in many countries is correct thrombocytopenia with platelet transfusion prior to CVC insertion, with the objective to mitigate the risk of serious pre or post procedural bleeding (23). The platelet count threshold recommended prior to CVC insertion varies significantly from country to country. In the UK the current threshold is $50 \times 10^{9} / 1$ (24), in Belgium the threshold is $30 \times 10^{9} / 1$ (25), in the United States (US) the threshold is $20 \times 10^{9} / 1$ (26) and in Germany the threshold is $10 \times 10^{9} / 1$ unless there are risk factors for bleeding (27). However it must be emphasized that platelet transfusion may be associated with adverse event such as rigors, fever, urticaria; serious sequelae are rare and include anaphylaxis, that can be life threatening $(23,28)$. In addition the requirement to administer platelet transfusions to correct thrombocytopenia prior to central line insertion may additionally delay the start of treatment, which may be time-critical in patient with acute leukemia, or in patient in intensive care.

Above all it remains unclear whether platelet transfusion in thrombocytopenic non-bleeding patients, despite improving the platelet count, reduce the incidence of clinically-important bleeding or improve other meaningful patient-oriented outcomes, such as mortality $(23,28)$. Patients may therefore be exposed to the risks of a platelet transfusion without any obvious clinical benefit. In a retrospective analysis, that included 193 consecutive adult patients receiving 604 CVC insertions, 93 cases had platelet count $\leq 20 \times 10^{9} / 1$ : The majority of bleedings were grade 1 , and $4 \%$ grade 2 , while no moderate, and no severe bleedings (grade 3 or 4) were reported (29) it must be emphasized, that in this series the number of pneumothorax was 6 on 604 (1\%), but in this series the CVCs were inserted without US-guidance. Our data demonstrate that in a large number of cancer patients: 1,867 underwent 2,187 US-guided insertional procedures no pneumothorax were reported, in addition, between the 110 thrombocytopenic cancer patients, that underwent US-guided CVC insertion, only one patient showed a local mild hematoma, without clinical significance, no patients of this series received prophylactic platelet transfusion to prevent bleeding of CVC insertional procedure. Other invasive procedures such as thoracentesis in thrombocytopenic cancer patients may be safety done under US-guidance, without prophylactic platelet transfusion as recently reported by our group (30), and other report confirm that US-guided CVC is a safe and highly successful modality also in liver disease patients with damaged coagulation (31). A prospective, randomized controlled trial powered to test the hypothesis of whether omitting forgoing platelet transfusion prior to US-guided CVC insertion leads to an equal occurrence of clinically relevant bleeding complications in patients with thrombocytopenia is currently recruiting (32). In conclusion, we agree with the review article reported in this journal that stated it is strongly recommended to use real-time US-guidance for central venous access (33) and we believe that US-guidance of CVC insertion procedures makes the difference in safety, also in thrombocytopenic patients avoiding prophylactic platelet transfusion.

\section{Acknowledgements}

Not applicable.

\section{Funding}

No funding was received.

\section{Availability of data and materials}

The datasets generated and/or analyzed during the present study are not publicly available due to the hospital's privacy regulations but are available from the corresponding author on reasonable request.

\section{Authors' contributions}

LC designed the study, performed the experiments and wrote the paper. CC collected and analyzed the data, and wrote the paper. CDN analyzed the data and wrote the paper. EO, IT and MA performed the experiments and wrote the paper. All authors read and approved the final manuscript.

\section{Ethics approval consent to participate}

All of the patients included in the present retrospective study previously signed informed consent before undergoing the procedure and provided informed consent for the use of their data for clinical research.

\section{Patient consent for publication}

Patients gave their written informed consent prior to their inclusion in this report.

\section{Competing interests}

The authors declare that they have no competing interests.

\section{References}

1. McGee DC and Gould MK: Preventing complications of central venous catheterisation. N Engl J Med 348: 1123-1133, 2003.

2. Cortelezzia A, Fracchiolla NS, Maisonneuve P, Moia M, Luchesini C, Ranzi ML, Monni P, Pasquini MC and Lambertenghi-Deliliers G: Central venous catheter-related complications in patients with hematological malignancies. A retrospective analysis of risk factors and prophylactic measures. Leuk Lymphoma 44: 1495-1501, 2003.

3. Bernard RW and Sthal WM: Subclavian vein catheterizations: A prospective study. I. Non-infectious complications. Ann Surg 173: 184-190, 1971.

4. Sznajder JI, Zveibil FR, Bitterman H, Weiner P and Bursztein S: Central vein catheterization. Failure and complication rates by three percutaneous approaches. Arch Intern Med 146: 259-261, 1986.

5. Defalque RJ: Percutaneous catheterization of the internal jugular vein. Anesth Analg 53: 116-121, 1974 
6. Mallory DL, McGee WT, Shawker TH, Brenner M, Bailey KR, Evans RG, Parker MM, Farmer JC and Parillo JE: Ultrasound guidance improves the success rate of internal jugular vein cannulation: A prospective, randomized trial. Chest 98: 157-160, 1990.

7. Troianos CA, Jobes DR and Ellison N: Ultrasound-guided cannulation of the internal jugular vein. A prospective, randomized study. Anesth Analg 72: 823-826, 1991.

8. Denys BG, Uretsky BF and Reddy PS: Ultrasound-assisted cannulation of the internal jugular vein. A prospective comparison to the external landmark-guided technique. Circulation 87: $1557-1562,1993$.

9. Slama M, Novara A, Safavian A, Ossart M, Safar M and Fagon JY: Improvement of internal jugular vein cannulation using an ultrasound-guided technique. Intensive Care Med 23: 916-919, 1997.

10. Teichgräber UK, Benter T, Gebel M and Mann MP: A sonographically guided technique for central venous access. AJR Am J Roentgenol 169: 731-733, 1997.

11. Nadig C, Leidig M, Schmiedeke T and Höffken B: The use of ultrasound for the placement of dialysis catheters. Nephrol Dial Transplant 13: 978-981, 1998

12. Hayashi $\mathrm{H}$ and Amano $\mathrm{M}$ : Does ultrasound imaging before puncture facilitate internal jugular vein cannulation? Prospective randomized comparison with landmark-guided puncture in ventilated patients. J Cardiothorac Vasc Anesth 16: 572-575, 2002.

13. Leung J, Duffy $M$ and Finckh A: Real-time ultrasonographically-guided internal jugular vein catheterization in the emergency department increases success rates and reduces complications: A randomized, prospective study. Ann Emerg Med 48: 540-547, 2006.

14. Karakitsos D, Labropoulos N, De Groot E, Patrianakos AP, Kouraklis G, Poularas J, Samonis G, Tsoutsos DA, Konstadoulakis MM and Karabinis A: Real-time ultrasound-guided catheterisation of the internal jugular vein: A prospective comparison with the landmark technique in critical care patients. Crit Care 10: R162, 2006.

15. Randolph AG, Cook DJ, Gonzales CA and Pribble CG: Ultrasound guidance for placement of central venous catheters: A meta-analysis of the literature. Crit Care Med 24: 2053-2058, 1996.

16. Hind D, Calvert N, McWilliams R, Davidson A, Paisley S, Beverley $\mathrm{C}$ and Thomas S: Ultrasonic locating devices for central venous cannulation: Meta-analysis. BMJ 327: 361, 2003.

17. Cavanna L, Civardi G, Vallisa D, Di Nunzio C, Cappucciati L, Bertè R, Cordani MR, Lazzaro A, Cremona G, Biasini C, et al: Ultrasound-guided central venous catheterization in cancer patients improves the success rate of cannulation and reduces mechanical complications: A prospective observational study of 1,978 consecutive catheterizations. World J Surg Oncol 8: 91, 2010.

18. Cavanna L, Cordani MR, Biasini C, Di Nunzio C, Monfredo M, Stroppa E, Muroni M, Ambroggi M, Muroni L, Di Cicilia R, et al: Ultrasound-guided central venous catheterization for home parenteral nutrition and hydratation in advanced incurable cancer patients: Results of a prospective observational study. World J Oncol 2: 238-244, 2011.

19. Schiffer CA, Bohlke K, Delaney M, Hume H, Magdalinski AJ, McCullough JJ, Omel JL, Rainey JM, Rebulla P, Rowley SD, et al: Platelet transfusion for patients with cancer: American society of clinical oncology clinical practice guideline update. J Clin Oncol 36: 283-299, 2018
20. U.S. Department of Health and Human Services. Common terminology criteria for adverse events (CTCAE), Version 4.03, 2010.

21. Biffi R, de Braud F, Orsi F, Pozzi S, Mauri S, Goldhirsch A, Nolè F and Andreoni B: Totally implantable central venous access ports long-term chemotherapy. A prospective study analyzing complications and costs of 333 devices with a minimum follow-up of 180 days. Ann Oncol 9: 767-773, 1998.

22. Callum KG and Whimster F: Interventional vascular radiology and interventional neurovascular radiology. a report of the national confidential enquiry into perioperative deaths. London, NCEPOD, 2000

23. Estcourt LJ, Desborough M, Hopewell S, Doree C and Stanworth SJ: Comparison of different platelet transfusion thresholds prior to insertion of central lines in patients with thrombocytopenia. Cochrane Database Syst Rev: CD011771, 2015.

24. British Committee for Standards in Haematology, Blood Transfusion Task Force: Guidelines for the use of platelet transfusions. Br J Haematol 122: 10-23, 2003.

25. Bosly A, Muylle L, Noens L, Pietersz R, Heim D, Hübner R, Selleslag D, Toungouz M, Ferrant A and Sondag D: Guidelines for the transfusion of platelets. Acta Clin Belg 62: 36-47, 2007.

26. Kaufman RM, Djulbegovic B, Gernsheimer T, Kleinman S, Tinmouth AT, Capocelli KE, Cipolle MD, Cohn CS, Fung MK, Grossman BJ, et al: Platelet transfusion: A clinical practice guideline from the AABB. Ann Intern Med 162: 205-313, 2015.

27. The Board of the German Medical Association on the recommendation of the Scientific Advisory Board. Platelet transfusion. Trans Med Hemother 36, 2009.

28. Castaman G and Pieri L: Management of thrombocytopenia in cancer. Thromb Res 164 (Suppl 1): S89-S93, 2018.

29. Zeidler K, Arn K, Senn O, Schanz U and Stussi G: Optimal preprocedural platelet transfusion threshold for central venous catheter insertions in patients with thrombocytopenia. Transfusion 51: 2269-2276, 2011

30. Orlandi E, Citterio C, Seghini P, Di Nunzio C, Mordenti P and Cavanna L: Thoracentesis in advanced cancer patients with severe thrombocytopenia: Ultrasound guide improves safety and reduces bleeding risk. Clin Respir J 12: 1747-1752, 2018.

31. Singh SA, Sharma S, Singh A, Singh AK, Sharma U and Bhadoria AS: The safety of ultrasound guided central venous cannulation in patients with liver disease. Saudi J Anaesth 9: 155-160, 2015.

32. van de Weerdt EK, Biemond BJ, Zeerleder SS, van Lienden KP, Binnekade JM and Vlaar APJ: Study collaborative: Prophylactic platelet transfusion prior to central venous catheter placement in patients with thrombocytopenia: Study protocol for a randomised controlled trial. Trials 19: 127, 2018.

33. Dietrich CF, Horn R, Morf S, Chiorean L, Dong Y, Cui XW, Atkinson NS and Jenssen C: Ultrasound-guided central vascular interventions, comments on the European federation of societies for ultrasound in medicine and biology guidelines on interventional ultrasound. J Thorac Dis 8: E851-E868, 2016.

This work is licensed under a Creative Commons Attribution-NonCommercial-NoDerivatives 4.0 International (CC BY-NC-ND 4.0) License. 